\section{Padrão de atividade física em um dia típico de adultos de Niterói, Rio de Janeiro, Brasil: resultados da Pesquisa de Nutrição, Atividade Física e Saúde (PNAFS)}

\author{
Patterns of physical activity during a typical day for \\ adults in Niterói, Rio de Janeiro State, Brazil: the \\ Nutrition, Physical Activity, and Health Survey \\ (PNAFS)
}

\begin{abstract}
${ }^{1}$ Departamento de Nutrição Social, Universidade Federal Fluminense, Niterói, Brasil. 2 Escola Nacional de Ciências Estatísticas, Instituto

Brasileiro de Geografia e Estatística, Rio de Janeiro, Brasil.

Correspondência

L. A. Anjos

Laboratório de Avaliação Nutricional e Funcional, Departamento de Nutrição Social, Universidade Federal Fluminense.

Rua Mário Santos Braga 30, Niterói, RJ 24020-140, Brasil. anjos@ensp.fiocruz.br
\end{abstract}

\begin{abstract}
The current study aimed to assess patterns of typical daily physical activity in a probability sample $(N=1,689)$ of adults in Niterói, Rio de Janeiro State, Brazil, using 24-hour recall. The sample was designed in three stages (census enumeration area-CEA, household within the CEA, and an adult selected with equal probability among all eligible adults in the household). Physical activities were classified as light, moderate, or vigorous and grouped in household chores, occupational, leisure-time, and commuting. Men performed more moderate and vigorous activities during significantly more time $(91.2 \pm 5.6$ and $8.2 \pm 1.4$ minutes, respectively; mean $\pm S D$ ) as compared to women $(68.5 \pm 2.7$ and $2.9 \pm 0.4$ minutes, respectively). The proportions of adults that reached the recommended 30 minutes of moderate/vigorous physical activity per day were 63.8\% (men) and 55.9\% (women). Household chores for women and leisure-time activity for men contributed the most to total moderate/vigorous physical activity. In conclusion, most adults in Niterói are physically active.
\end{abstract}

Motor Activity; Activities of Daily Living; Adult Health
Luiz Antonio dos Anjos 1

Thomaz Barcellos Côrtes Barbosa ${ }^{1}$

Vivian Wahrlich 1

Mauricio Teixeira Leite de Vasconcellos 2

\section{Introdução}

A inatividade física é considerada pela Organização Mundial da Saúde (OMS) como um dos principais fatores de risco para o desenvolvimento de diversas doenças crônicas não transmissíveis, como doenças cardiovasculares, diabetes, osteoporose e alguns tipos de câncer ${ }^{1}$. A manutenção de uma vida fisicamente ativa reduz substancialmente o risco para tais doenças e proporciona diversos benefícios para saúde, como redução da adiposidade corporal; melhora da saúde cardiovascular, óssea e muscular; diminuição da ansiedade e depressão; dentre outros 2.

A recomendação hegemônica atual preconiza que todos os adultos realizem pelo menos 30min de atividade física aeróbia em intensidade moderada cinco dias por semana, ou 20min de atividade pesada três dias por semana para promoção e manutenção da saúde ${ }^{3}$. Para aqueles que desejam melhorar a aptidão física ou reduzir ainda mais os riscos para doenças crônicas e mortalidade associadas à inatividade física, a recomendação é maior do que essa quantidade mínima. Para a manutenção da massa corporal, recomenda-se cerca de 60 a $90 \mathrm{~min}$ de atividade física moderada por dia 4 .

Diversos métodos e equipamentos (calorimetria, marcadores fisiológicos, inquéritos, instrumentos mecânicos ou eletrônicos) são utilizados para avaliar a atividade física e, assim, 
estabelecer sua relação com a saúde e a doença em estudos epidemiológicos ou determinar o padrão de atividade física populacional 5,6,7. Entretanto, nem todos possuem uma boa acurácia em sua estimativa, e quando têm, apresentam alto custo ou dificuldade de aplicação em larga escala 6. Um método amplamente utilizado em estudos epidemiológicos é o questionário de atividade física pelo seu baixo custo e sua fácil aplicação ${ }^{6}$. Até recentemente, os estudos avaliavam a atividade física por meio de questionários utilizando somente informações sobre atividade física de lazer 8,9. No entanto, há algum tempo é reconhecido que a medição somente da atividade física de lazer leva à subestimação da atividade física total, principalmente em pessoas com ocupações mais pesadas ${ }^{10}$. Assim, estudos que só investigam a atividade física de lazer podem subestimar o padrão de atividade física, pois pessoas consideradas "sedentárias" podem possuir um nível de atividade física adequado para obter os benefícios para saúde advindos de outros tipos de atividade, não só a ocupacional, como também a de deslocamento ou doméstica, que pode ter grande significado em certos grupos populacionais 11. Nesse sentido, desenvolveu-se um questionário que avaliaria todos os domínios da atividade física (lazer, ocupacional, deslocamento, doméstica), o chamado IPAQ (Questionário Internacional de Atividade Física), sugerido para uso internacional 12. Entretanto, estudos de sua validação têm demonstrado superestimativa e baixa correlação com a atividade física medida de forma objetiva 13,14,15,16,17.

Uma alternativa é o recordatório de 24 horas, instrumento largamente utilizado em pesquisas epidemiológicas para captar a ingestão de alimentos do dia anterior, que pode obter, concomitantemente, as atividades realizadas no dia anterior 18. Esse procedimento facilita a lembrança dos alimentos ingeridos, além de permitir a obtenção detalhada da duração e intensidade de todas as atividades realizadas, dados importantes para estimar o gasto energético do indivíduo no período 18 , informação recomendada pela OMS para o estabelecimento das recomendações energéticas ${ }^{19}$. O recordatório de atividade física de $24 \mathrm{~h}$ (R24h) já foi validado contra outros questionários ou método objetivo (acelerômetro) $20 \mathrm{e}$ é, frequentemente, usado em estudos de sazonalidade 21,22. Dessa forma, o objetivo desta pesquisa foi avaliar a atividade física de um dia típico, em todos os domínios, e verificar se os adultos do Município de Niterói, Rio de Janeiro, realizam os 30min de atividade física moderada atualmente recomendados.

\section{Métodos}

Os dados do presente estudo são da Pesquisa de Nutrição, Atividade Física e Saúde (PNAFS), inquérito domiciliar realizado entre janeiro e dezembro de 2003, em uma amostra probabilística da população adulta ( $\geq 20$ anos de idade) residente no Município de Niterói 23. A amostra da PNAFS foi desenhada em três estágios: setor censitário, domicílio particular permanente (DPP) e um adulto ( $\geq 20$ anos) no domicílio selecionado. Os setores do Censo Demográfico 200024 (primeiro estágio) foram ordenados por renda média domiciliar, o que, associado à seleção sistemática dos setores, correspondeu a uma estratificação implícita por renda que conduziu a uma amostra composta, para cada quinto de renda, por 22 setores selecionados com probabilidade proporcional ao seu número de DPP. No segundo estágio, em cada um dos 110 setores selecionados foi feita uma operação de listagem de domicílios para gerar o cadastro com base no qual foram selecionados, com equiprobabilidade, endereços para serem sequencialmente visitados até que fossem obtidas 16 entrevistas realizadas, dentro de um procedimento de amostragem inversa 25 , semelhante ao utilizado na Pesquisa Mundial de Saúde no Brasil 26. Dessa forma, a amostra é composta por 1.760 domicílios com entrevistas realizadas. Em cada domicílio, um adulto foi selecionado para participar do estudo (terceiro estágio), com equiprobabilidade entre os adultos elegíveis do domicílio. Um adulto era elegível se não apresentasse qualquer condição patológica que pudesse influenciar o metabolismo, se não fizesse uso de algum medicamento que alterasse a frequência cardíaca ou o metabolismo, ou ainda se não estivesse fazendo dieta para emagrecimento. Uma visita prévia a cada domicílio selecionado foi realizada para: (1) explicar os objetivos do estudo e a sistemática de sua operação de coleta; (2) solicitar o consentimento para a pesquisa; e (3) marcar data e horário para o início da pesquisa. Pesos amostrais iniciais foram obtidos pelo inverso da probabilidade de inclusão do adulto (produto das probabilidades de inclusão em cada estágio de seleção), que foram calibrados para gerarem estimativas coerentes com os totais populacionais por sexo e grupo etário, usando o Integrated Household Weighting System 26,27, como descrito anteriormente 23,28 .

As medidas de massa corporal e estatura foram obtidas no próprio domicílio como descrito previamente 23 . Com o adulto selecionado de cada domicílio, realizaram-se recordatórios de 24h: alimentar 28 e de atividade física (R24h). As entrevistas para o R24h visavam a obter, retrospectivamente, tudo o que o indivíduo havia feito 
nas $24 \mathrm{~h}$ anteriores. Caso o dia não houvesse sido típico, do ponto de vista de atividades, repetiamse os procedimentos numa outra oportunidade.

As atividades do R24h, junto com suas durações, foram usadas para descrever as atividades cotidianas realizadas pela população. As atividades foram recordadas em períodos de 15 a 30min ou por blocos de tempo (dependendo da duração da atividade), anotadas pelo pesquisador em papel e posteriormente transcritas para planilhas eletrônicas. Embora a padronização fosse anotar as atividades em intervalos de 15 a 30min, alguns recordatórios apresentaram relatos de diferentes atividades (até quatro) num intervalo de tempo superior a 30min, mas sem especificação do tempo de realização de cada uma das atividades. Isso aconteceu devido à dificuldade de alguns indivíduos em recordar com precisão as atividades realizadas. Nesses casos, o critério utilizado foi dividir o tempo em partes iguais para as atividades relatadas ou usar o tempo da mesma atividade realizada pelo próprio indivíduo em outro momento do dia. Caso não houvesse essa informação, usava-se o tempo médio da atividade na amostra. Por exemplo, fazendo a média de tempo gasto para jantar daqueles indivíduos que relataram ter feito esta refeição num tempo definido, obteve-se uma duração média de 30min. Portanto, se o indivíduo relatou ter estudado e jantado num tempo de 200min, pressupôs-se que o mesmo gastou 30min jantando e passou os restantes 170 min estudando. Esses tempos médios foram utilizados para as seguintes atividades: escovar os dentes = $5 \mathrm{~min}$; arrumar-se $=25 \mathrm{~min}$; refeições $=30 \mathrm{~min}$ e tomar banho $=25 \mathrm{~min}$.

Definido o tempo de duração de cada atividade relatada, estabeleceu-se a intensidade das atividades em múltiplos do metabolismo de repouso expresso como equivalente metabólico (MET), usando o Compêndio de Atividades proposto por Ainsworth et al. 29. A intensidade das atividades desempenhadas pelos indivíduos durante o tempo acordado foram classificadas segundo o modelo proposto por Pate et al. $30 \mathrm{em}$ : leve (<3 METs), moderada (3-5,9 METs) e pesada ( $\geq 6$ METs). Dessa maneira, foi possível identificar o período de permanência nos diferentes níveis de atividade e verificar se os mesmos realizaram o mínimo de atividade atualmente proposto (30min de atividade física em intensidade moderada/pesada). Para calcular o percentual de indivíduos que atingiram os 30min diários de atividade física moderada/pesada foram computadas somente as atividades realizadas por um período mínimo de 10min. Dessa forma, o indivíduo pode ter feito uma única sessão de $30 \mathrm{~min}$ ou duas sessões de $15 \mathrm{~min}$ ou três de $10 \mathrm{~min}$. As atividades durante o tempo acordado foram agrupadas em quatro domínios: (1) atividades domésticas; (2) atividades ocupacionais; (3) atividades de lazer (incluindo o exercício físico realizado para fins de condicionamento); e (4) deslocamentos. Além das características das atividades, computou-se a prevalência de indivíduos que relataram realizar exercício para condicionamento físico ou esporte no lazer.

A análise dos dados incluiu o cálculo de média, erro padrão e intervalo de $95 \%$ de confiança (IC95\%) para as variáveis contínuas. Todas as análises foram realizadas com pesos amostrais calibrados, usando estimação por domínio (DOMAIN), com os procedimentos surveymeans e surveyfreq do SAS versão 9.2 (SAS Inst., Cary, Estados Unidos), que trata adequadamente desenhos de amostra complexa. A significância das diferenças entre médias e proporções por sexo e faixa etária foi estabelecida quando não havia interseção entre os IC95\% correspondentes.

\section{Resultados}

Foi possível obter dados completos com 1.727 indivíduos, já que 22 se recusaram a responder ao recordatório e 11 não conseguiram dar informações detalhadas o suficiente para as análises. Foram excluídos 38 indivíduos por apresentarem $\mathrm{R} 24 \mathrm{~h}$ realizado em dias atípicos (e não aceitarem repetir). Portanto, a presente pesquisa compreendeu um total de 1.689 indivíduos, sendo 1.178 mulheres e 511 homens. Com a expansão dos dados da amostra para a população, as estimativas representam os 324.671 habitantes adultos do Município de Niterói, sendo 178.785 mulheres e 145.886 homens.

A idade máxima dos participantes foi 86,9 (mulheres) e 81,9 anos (homens) com média ( \pm erro padrão) de 45,3 $\pm 0,6$ e $43,0 \pm 0,8$ anos para mulheres e homens, respectivamente (Tabela 1).

As mulheres passaram, em média, aproximadamente $14 \mathrm{~h}$ e $54 \mathrm{~min}(839,9 \pm 3,9 \mathrm{~min})$ do período em que permaneceram acordadas $(92,6 \%)$ em atividades leves (<3 METs) e somente $1 \mathrm{~h}$ e $11 \mathrm{~min}$ (7,4\%) em atividades moderadas $(68,5 \mathrm{~min})$ ou pesadas (2,9min; Tabela 2). Os homens de todas as idades mostraram-se significativamente mais ativos fisicamente do que as mulheres, pois permaneceram, em média, $1 \mathrm{~h}$ e $40 \mathrm{~min}$ (10\% do tempo acordado) em atividades de intensidade moderada $(91,2 \mathrm{~min})$ a pesada $(8,2 \mathrm{~min})$, mas o tempo em atividades leves ( $14 \mathrm{~h}$ e $55 \mathrm{~min}$ ) foi bem próximo do das mulheres. A comparação entre sexo para cada faixa etária não mostrou diferença significativa. Com o avançar da idade, as mulheres apresentaram diminuição das atividades moderadas/pesadas e aumento do tempo dedicado 
Tabela 1

Estimativa de médias e erro padrão (EP) da idade e medidas antropométricas da população adulta ( $\geq 20$ anos) de Niterói, Rio de Janeiro, Brasil, segundo sexo e faixa etária. Pesquisa de Nutrição, Atividade Física e Saúde (PNAFS), 2003.

\begin{tabular}{|c|c|c|c|c|c|c|}
\hline \multirow[t]{3}{*}{ Idade e antropometria } & \multirow{3}{*}{$\begin{array}{l}\text { Todas as } \\
\text { idades } \\
\text { Média } \pm \text { EP } \\
\text { (IC95\%) }\end{array}$} & \multicolumn{5}{|c|}{ Faixa etária (anos) } \\
\hline & & $20-29,9$ & $30-39,9$ & $40-49,9$ & $50-59,9$ & $\geq 60$ \\
\hline & & $\begin{array}{l}\text { Média } \pm \text { EP } \\
\text { (IC95\%) }\end{array}$ & $\begin{array}{l}\text { Média } \pm \text { EP } \\
\text { (IC95\%) }\end{array}$ & $\begin{array}{l}\text { Média } \pm \text { EP } \\
\text { (IC95\%) }\end{array}$ & $\begin{array}{l}\text { Média } \pm \text { EP } \\
\text { (IC95\%) }\end{array}$ & $\begin{array}{l}\text { Média } \pm \text { EP } \\
\text { (IC95\%) }\end{array}$ \\
\hline \multicolumn{7}{|l|}{ Mulheres } \\
\hline $\mathrm{n}$ & 178.785 & 39.564 & 37.939 & 36.603 & 26.205 & 38.474 \\
\hline Idade (anos) & $\begin{array}{c}45,3 \pm 0,6 \\
(44,1 ; 46,6)\end{array}$ & $\begin{array}{l}24,8 \pm 0,2 a \\
(44,4 ; 25,1)\end{array}$ & $\begin{array}{l}35,5 \pm 0,2 a \\
(35,1 ; 35,8)\end{array}$ & $\begin{array}{l}45,3 \pm 0,2 a \\
(44,9 ; 45,7)\end{array}$ & $\begin{array}{l}54,3 \pm 0,2 a \\
(54,0 ; 57,7)\end{array}$ & $\begin{array}{l}70,0 \pm 0,5 a \\
(69,0 ; 71,1)\end{array}$ \\
\hline Massa corporal $(\mathrm{kg})$ * & $\begin{array}{c}63,8 \pm 0,4 \\
(63,1 ; 64,6)\end{array}$ & $\begin{array}{c}61,3 \pm 0,8 \mathrm{ab} \\
(59,8 ; 62,8)\end{array}$ & $\begin{array}{c}63,7 \pm 0,8 \\
(62,0 ; 65,4)\end{array}$ & $\begin{array}{l}65,4 \pm 0,7 a \\
(63,9 ; 66,8)\end{array}$ & $\begin{array}{c}67,2 \pm 0,9 b c \\
(65,4 ; 69,0)\end{array}$ & $\begin{array}{l}62,8 \pm 0,7 c \\
(61,3 ; 64,2)\end{array}$ \\
\hline Estatura $(\mathrm{cm})$ * & $\begin{array}{c}158,6 \pm 0,2 \\
(158,1 ; 159,0)\end{array}$ & $\begin{array}{l}161,5 \pm 0,3 a b c \\
(160,8 ; 162,2)\end{array}$ & $\begin{array}{l}160,2 \pm 0,4 d \\
(159,3 ; 161,1)\end{array}$ & $\begin{array}{l}158,6 \pm 0,4 \text { ae } \\
(157,9 ; 159,4)\end{array}$ & $\begin{array}{l}157,5 \pm 0,4 b d \\
(156,7 ; 158,3)\end{array}$ & $\begin{array}{c}154,5 \pm 0,5 \text { cde } \\
(153,5 ; 155,5)\end{array}$ \\
\hline \multicolumn{7}{|l|}{ Homens } \\
\hline $\mathrm{n}$ & 145.886 & 36.609 & 33.309 & 30.313 & 21.043 & 24.612 \\
\hline Idade (anos) & $\begin{array}{c}43,0 \pm 0,8 \\
(41,5 ; 44,5)\end{array}$ & $\begin{array}{l}25,0 \pm 0,2 a \\
(24,5 ; 25,5)\end{array}$ & $\begin{array}{l}35,1 \pm 0,3 a \\
(34,5 ; 35,6)\end{array}$ & $\begin{array}{l}44,7 \pm 0,3 a \\
(44,0 ; 45,3)\end{array}$ & $\begin{array}{l}54,5 \pm 0,3 a \\
(53,8 ; 55,1)\end{array}$ & $\begin{array}{l}68,7 \pm 0,7 \mathrm{a} \\
(67,2 ; 70,1)\end{array}$ \\
\hline Massa corporal $(\mathrm{kg})$ * & $\begin{array}{c}75,2 \pm 0,7 \\
(73,9 ; 76,5)\end{array}$ & $\begin{array}{l}75,1 \pm 1,1 \mathrm{a} \\
(73,0 ; 77,2)\end{array}$ & $\begin{array}{l}77,9 \pm 1,5 b \\
(75,0 ; 80,8)\end{array}$ & $\begin{array}{l}77,0 \pm 1,4 c \\
(74,2 ; 79,7)\end{array}$ & $\begin{array}{c}75,7 \pm 1,9 \mathrm{dc} \\
(71,8 ; 79,6)\end{array}$ & $\begin{array}{c}68,8 \pm 1,1 \mathrm{abcd} \\
(66,5 ; 71,1)\end{array}$ \\
\hline Estatura $(\mathrm{cm})$ * & $\begin{array}{c}171,8 \pm 0,4 \\
(171,1 ; 172,6)\end{array}$ & $\begin{array}{l}175,5 \pm 0,7 a b \\
(174,1 ; 176,9)\end{array}$ & $\begin{array}{c}173,7 \pm 0,8 c \\
(172,1 ; 175,3)\end{array}$ & $\begin{array}{l}170,6 \pm 0,7 \text { ac } \\
(169,1 ; 172,0)\end{array}$ & $\begin{array}{l}170,2 \pm 1,0 b \\
(168,1 ; 172,3)\end{array}$ & $\begin{array}{l}166,8 \pm 0,6 \mathrm{ac} \\
(165,6 ; 168,0)\end{array}$ \\
\hline \multicolumn{7}{|l|}{ Todos } \\
\hline $\mathrm{N}$ & 324.671 & 76.173 & 71.248 & 66.916 & 47.248 & 63.086 \\
\hline Idade (anos) & $\begin{array}{c}44,3 \pm 0,5 \\
(43,3 ; 45,3)\end{array}$ & $\begin{array}{l}24,9 \pm 0,2 a \\
(24,6 ; 25,2)\end{array}$ & $\begin{array}{l}35,3 \pm 0,2 a \\
(34,9 ; 35,6)\end{array}$ & $\begin{array}{l}45,0 \pm 0,2 a \\
(44,7 ; 45,4)\end{array}$ & $\begin{array}{l}54,4 \pm 0,2 a \\
(54,0 ; 54,7)\end{array}$ & $\begin{array}{l}69,5 \pm 0,4 a \\
(68,7 ; 70,3)\end{array}$ \\
\hline Massa corporal (kg) & $\begin{array}{c}68,9 \pm 0,4 \\
(68,1 ; 69,7)\end{array}$ & $\begin{array}{l}67,9 \pm 0,7 a \\
(66,5 ; 69,3)\end{array}$ & $\begin{array}{l}70,4 \pm 0,9 b \\
(68,6 ; 72,1)\end{array}$ & $\begin{array}{l}70,6 \pm 0,8 c \\
(69,0 ; 72,2)\end{array}$ & $\begin{array}{c}71,0 \pm 1,0 \mathrm{dc} \\
(68,9 ; 73,1)\end{array}$ & $\begin{array}{c}65,1 \pm 0,6 \mathrm{abcd} \\
(63,9 ; 66,4)\end{array}$ \\
\hline Estatura $(\mathrm{cm})$ & $\begin{array}{c}164,5 \pm 0,3 \\
(164,0 ; 165,0)\end{array}$ & $\begin{array}{l}168,2 \pm 0,5 \mathrm{ab} \\
(167,2 ; 169,3)\end{array}$ & $\begin{array}{c}166,5 \pm 0,5 c \\
(165,4 ; 167,6)\end{array}$ & $\begin{array}{l}164,1 \pm 0,5 \mathrm{ad} \\
(163,1 ; 165,0)\end{array}$ & $\begin{array}{l}163,2 \pm 0,7 b c \\
(161,7 ; 164,6)\end{array}$ & $\begin{array}{c}159,3 \pm 0,6 \mathrm{bcd} \\
(158,1 ; 165,0)\end{array}$ \\
\hline
\end{tabular}

* Todas as estimativas de média (total e por idade) são significativamente (5\%) diferentes entre os sexos.

Nota: estimativas de médias de uma mesma linha com a mesma letra são significativamente (5\%) diferentes entre si.

a atividades leves. Tendência semelhante foi observada para homens, mas com menos diferenças significativas entre as faixas etárias. Porém, as atividades pesadas aumentam até os 40 anos, para um posterior declínio. Os homens na faixa etária dos 30-39,9 anos apresentaram um tempo maior nas atividades pesadas do que os com idade superior a 60 anos, o que também ocorreu nas atividades moderadas para mulheres e homens.

A prevalência de adultos que reportaram realizar exercícios para condicionamento físico ou esporte no lazer foi de 80,7\% (IC95\%: 77,7\%$83,7 \%$ ) sendo significativamente maior para mulheres (84,9\%; IC95\%: 81,9\%-87,9\%) do que para homens (75,5\%; IC95\%: 70,5\%-80,6\%).

Ao avaliar a prática de atividade física moderada/pesada ( $\geq 3$ METs) da população adulta de Niterói durante as $24 \mathrm{~h}$, verificou-se que $59,5 \%$ dos indivíduos realizaram pelo menos os 30min de atividade física atualmente recomendados. Comparando homens e mulheres, observou-se um percentual maior, mas não significativo, para homens (63,8\% vs. 55,9\%; Tabela 3). Apesar da tendência de redução na proporção de homens e mulheres que alcançavam a recomendação com o avançar da idade, não houve diferença significativa entre as faixas etárias. Tanto para mulheres quanto homens, a maior proporção dos que alcançavam a recomendação foi na faixa etária de 30-39,9 anos (73,5\% e 65,6\%, respectivamente).

Analisando-se o domínio no qual foi realizada a atividade moderada/pesada na população adulta de Niterói, observou-se que as atividades domiciliares foram as que mais contribuíram (34,1\%; IC95\%: $30,7 \%-37,4 \%)$ no total, seguidas das atividade de lazer ativo e exercício físi- 
Estimativa de médias, erro padrão (EP) do tempo (minutos) acordado por sexo e faixa etária, segundo intensidade da atividade durante 24 horas em um dia típico na população adulta ( $\geq 20$ anos) de Niterói, Rio de Janeiro, Brasil. Pesquisa de Nutrição, Atividade Física e Saúde (PNAFS), 2003.

\begin{tabular}{|c|c|c|c|c|c|c|}
\hline \multirow[t]{3}{*}{ Intensidade (METs) } & \multirow[t]{2}{*}{ Todas as idades } & \multicolumn{5}{|c|}{ Faixa etária (anos) } \\
\hline & & $20-29,9$ & $30-39,9$ & $40-49,9$ & $50-59,9$ & $\geq 60$ \\
\hline & $\begin{array}{c}\text { Média } \pm \text { EP } \\
\text { (IC95\%) }\end{array}$ & $\begin{array}{c}\text { Média } \pm \text { EP } \\
\text { (IC95\%) }\end{array}$ & $\begin{array}{l}\text { Média } \pm \text { EP } \\
\text { (IC95\%) }\end{array}$ & $\begin{array}{c}\text { Média } \pm \text { EP } \\
\text { (IC95\%) }\end{array}$ & $\begin{array}{c}\text { Média } \pm \text { EP } \\
\text { (IC95\%) }\end{array}$ & $\begin{array}{c}\text { Média } \pm \text { EP } \\
\text { (IC95\%) }\end{array}$ \\
\hline \multicolumn{7}{|l|}{ Mulheres } \\
\hline Leve $(<3)$ & $\begin{array}{c}893,9 \pm 3,9 \\
(885,3 ; 902,4)\end{array}$ & $\begin{array}{c}855,9 \pm 8,2 a b c \\
(840,2 ; 871,6)\end{array}$ & $\begin{array}{l}866,7 \pm 8,5 d \\
(869,4 ; 904,0)\end{array}$ & $\begin{array}{l}899,0 \pm 8,6 a \\
(880,5 ; 917,6)\end{array}$ & $\begin{array}{l}923,7 \pm 8,8 \text { bd } \\
(907,1 ; 940,3)\end{array}$ & $\begin{array}{l}914,9 \pm 8,6 c \\
(897,6 ; 932,1)\end{array}$ \\
\hline Moderada $(3-5,9)$ & $\begin{array}{l}68,5 \pm 2,7 \text { * } \\
(61,9 ; 75,2)\end{array}$ & $\begin{array}{c}70,2 \pm 6,0 \\
(57,7 ; 82,6)\end{array}$ & $\begin{array}{l}83,4 \pm 6,0 \text { a } \\
(69,6 ; 97,2)\end{array}$ & $\begin{array}{l}74,8 \pm 6,4 b \\
(60,5 ; 89,2)\end{array}$ & $\begin{array}{c}64,5 \pm 6,7 \\
(52,1 ; 76,9)\end{array}$ & $\begin{array}{c}40,9 \pm 5,2 a b \\
(38,3 ; 59,6)\end{array}$ \\
\hline Pesada $(\geq 6)$ & $\begin{array}{c}2,9 \pm 0,4 \text { * } \\
(2,1 ; 3,7)\end{array}$ & $\begin{array}{c}6,0 \pm 1,1 a b \\
(3,5 ; 8,6)\end{array}$ & $\begin{array}{c}3,5 \pm 0,7 \mathrm{~cd} \\
(2,1 ; 4,9)\end{array}$ & $\begin{array}{l}2,4 \pm 0,7 \\
(1,0 ; 3,9)\end{array}$ & $\begin{array}{c}1,0 \pm 0,4 \text { ac } \\
(0,2 ; 1,6)\end{array}$ & $\begin{array}{c}1,0 \pm 0,5 \mathrm{bd} \\
(0,1 ; 1,8)\end{array}$ \\
\hline \multicolumn{7}{|l|}{ Homens } \\
\hline Leve $(<3)$ & $\begin{array}{c}895,7 \pm 7,0 \\
(881,2 ; 910,2)\end{array}$ & $\begin{array}{c}884,2 \pm 12,0 \\
(858,9 ; 909,4)\end{array}$ & $\begin{array}{c}880,1 \pm 16,7 \\
(846,8 ; 913,5)\end{array}$ & $\begin{array}{c}902,9 \pm 16,5 \\
(871,2 ; 934,5)\end{array}$ & $\begin{array}{c}922,4 \pm 18,4 \\
(896,6 ; 948,2)\end{array}$ & $\begin{array}{l}902,2 \pm 13,8 \\
(880,3 ; 924,0)\end{array}$ \\
\hline Moderada $(3-5,9)$ & $\begin{array}{l}91,2 \pm 5,6 \text { * } \\
(80,0 ; 102,3)\end{array}$ & $\begin{array}{c}93,0 \pm 9,8 \\
(72,8 ; 113,2)\end{array}$ & $\begin{array}{l}123,8 \pm 13,0 \\
(96,6 ; 151,0)\end{array}$ & $\begin{array}{l}100,6 \pm 14,0 \\
(73,3 ; 127,8)\end{array}$ & $\begin{array}{l}62,5 \pm 14,0 \\
(37,1 ; 87,9)\end{array}$ & $\begin{array}{l}57,2 \pm 10,2 \\
(39,1 ; 75,2)\end{array}$ \\
\hline Pesada $(\geq 6)$ & $\begin{array}{l}8,2 \pm 1,4 \text { * } \\
(5,8 ; 10,5)\end{array}$ & $\begin{array}{l}7,6 \pm 2,0 \\
(3,7 ; 11,5)\end{array}$ & $\begin{array}{c}12,7 \pm 2,9 a \\
(7,5 ; 17,8)\end{array}$ & $\begin{array}{l}10,8 \pm 4,2 \\
(2,1 ; 19,3)\end{array}$ & $\begin{array}{l}4,4 \pm 3,7 \\
(0,5 ; 8,3)\end{array}$ & $\begin{array}{c}2,8 \pm 1,8 a \\
(0,5 ; 5,2)\end{array}$ \\
\hline \multicolumn{7}{|l|}{ Todos } \\
\hline Leve $(<3)$ & $\begin{array}{l}894,7 \pm 4,0 a b \\
(886,7 ; 902,7)\end{array}$ & $\begin{array}{c}869,5 \pm 7,3 \\
(855,0 ; 883,9)\end{array}$ & $\begin{array}{c}883,6 \pm 8,7 \\
(866,3 ; 900,9)\end{array}$ & $\begin{array}{c}900,8 \pm 9,0 \\
(882,9 ; 918,6)\end{array}$ & $\begin{array}{l}923,1 \pm 7,4 a \\
(908,4 ; 937,8)\end{array}$ & $\begin{array}{l}909,9 \pm 7,1 b \\
(895,9 ; 924,0)\end{array}$ \\
\hline Moderada $(3-5,9)$ & $\begin{array}{c}78,7 \pm 3,3 \\
(72,2 ; 85,2)\end{array}$ & $\begin{array}{l}81,1 \pm 5,7 \text { a } \\
(69,8 ; 92,5)\end{array}$ & $\begin{array}{l}102,3 \pm 7,2 b \\
(87,9 ; 116,6)\end{array}$ & $\begin{array}{c}86,5 \pm 7,5 c \\
(71,6 ; 101,3)\end{array}$ & $\begin{array}{c}63,6 \pm 6,3 \\
(51,1 ; 76,1)\end{array}$ & $\begin{array}{c}52,2 \pm 4,9 \text { asc } \\
(42,4 ; 61,9)\end{array}$ \\
\hline Pesada $(\geq 6)$ & $\begin{array}{l}5,3 \pm 0,6 \\
(4,0 ; 6,5)\end{array}$ & $\begin{array}{c}6,8 \pm 1,2 a b \\
(4,5 ; 9,1)\end{array}$ & $\begin{array}{c}7,8 \pm 1,2 \mathrm{~cd} \\
(5,3 ; 10,3)\end{array}$ & $\begin{array}{l}6,2 \pm 2,0 \\
(2,1 ; 10,3)\end{array}$ & $\begin{array}{c}2,5 \pm 0,9 a c \\
(0,6 ; 4,3)\end{array}$ & $\begin{array}{c}1,7 \pm 0,5 \mathrm{bd} \\
(0,7 ; 2,7)\end{array}$ \\
\hline
\end{tabular}

METs: múltiplos do equivalente metabólico.

* Estimativas de médias significativamente (5\%) diferentes entre os sexos.

Nota: estimativas de médias de uma mesma linha com a mesma letra são significativamente (5\%) diferentes entre si.

co (31,9\%; IC95\%: 28,8\%-35,0\%), deslocamento (22,1\%; IC95\%: 19,6\%-24,5\%) e ocupacionais (11,9\%; IC95\%: 10,2-13,7\%). Em relação ao sexo, as análises indicaram que as mulheres preencheram $45 \%$ desse tempo com atividades domésticas, $29 \%$ em atividades de lazer ativo e exercício físico, $17 \%$ em deslocamentos e $9 \%$ em atividades ocupacionais (Figura 1). Já os homens apresentaram 35\% do tempo em atividades de lazer ativo e exercício físico, $22 \%$ em tarefas domésticas, $28 \%$ em deslocamentos e $15 \%$ em atividades ocupacionais. Entre as mulheres, foi observado predomínio das atividades domésticas em todas as faixas etárias, seguido pelo lazer ativo/exercício físico. Entre os homens, o predomínio foi do exercício físico e lazer ativo em todas as faixas etárias, exceto naqueles entre 40-49,9 anos. Esse padrão não se modificou quando foram analisados somente os indivíduos que alcançavam a recomendação de pelo menos de 30min de atividade física moderada/pesada.

\section{Discussão}

Revisões recentes sobre a evolução da pesquisa da prática de atividade física no Brasil documentaram evolução importante no número de estudos locais sobre o tema nas últimas décadas 31,32, mas são enfáticas na conclusão sobre a necessidade de padronização de instrumentos, critérios e definições na área, para que os resultados dos estudos possam ser comparados. $\mathrm{O}$ primeiro estudo de base populacional realizado em regiões brasileiras que obteve a informação sobre a prática de atividade física foi a Pesquisa de Padrões de Vida (PPV), em 1997, que revelou que $80 \%$ da população não praticavam exercício 
Tabela 3

Distribuição (\%) de realização de 30 minutos de atividade física em intensidade moderada/pesada ( $\geq 3 \mathrm{METs}$ ) durante 24 horas em um dia típico por faixa etária, segundo sexo da população adulta ( $\geq 20$ anos de idade) de Niterói, Rio de Janeiro, Brasil. Pesquisa de Nutrição, Atividade Física e Saúde (PNAFS), 2003.

\begin{tabular}{|c|c|c|c|c|c|c|}
\hline & \multirow[t]{2}{*}{ Todas as idades } & \multicolumn{5}{|c|}{ Faixa etária (anos) } \\
\hline & & $20-29,9$ & $30-39,9$ & $40-49,9$ & $50-59,9$ & $\geq 60$ \\
\hline \multicolumn{7}{|l|}{ Mulheres } \\
\hline n (\%) & $99.911(55,9)$ & $22.786(57,6)$ & $24.877(65,6)$ a & $19.997(54,6)$ & $12.565(48,0)$ a & $19.686(51,2)$ \\
\hline IC95\% & 53,$2 ; 59,6$ & 49,$6 ; 65,6$ & 58,$4 ; 72,8$ & 48,$4 ; 60,9$ & 40,$3 ; 55,6$ & 42,$1 ; 60,2$ \\
\hline \multicolumn{7}{|l|}{ Homens } \\
\hline n (\%) & $93.102(63,8)$ & $23.783(65,0)$ & $24.490(73,5)$ & $19.113(63,1)$ & $12.798(60,8)$ & $12.918(52,5)$ \\
\hline IC95\% & 58,$9 ; 68,8$ & 55,$8 ; 74,1$ & 65,$4 ; 81,6$ & 53,$3 ; 72,8$ & 47,$0 ; 74,7$ & 38,$5 ; 66,5$ \\
\hline \multicolumn{7}{|l|}{ Todos } \\
\hline$n(\%)$ & $193.013(59,5)$ & $46.569(61,1)$ & $49.367(69,3) a b c$ & $39,110(58,5)$ a & $25.363(53,7) b$ & $32.604(51,7) \mathrm{c}$ \\
\hline IC95\% & 56,$3 ; 62,6$ & 55,$2 ; 67,0$ & 64,$1 ; 74,5$ & 52,$9 ; 64,0$ & 46,$0 ; 61,3$ & 43,$9 ; 59,4$ \\
\hline
\end{tabular}

Nota: estimativas de porcentagens de uma mesma linha com a mesma letra são significativamente (5\%) diferentes entre si.

Figura 1

Distribuição (\%) do tempo realizando atividade física moderada/pesada ( $\geq 3 \mathrm{METs}$ ) durante o tempo acordado em um dia típico por domínio, segundo sexo e faixa etária da população adulta ( $\geq 20$ anos de idade) de Niterói, Rio de Janeiro, Brasil. Pesquisa de Nutrição, Atividade Física e Saúde (PNAFS), 2003.
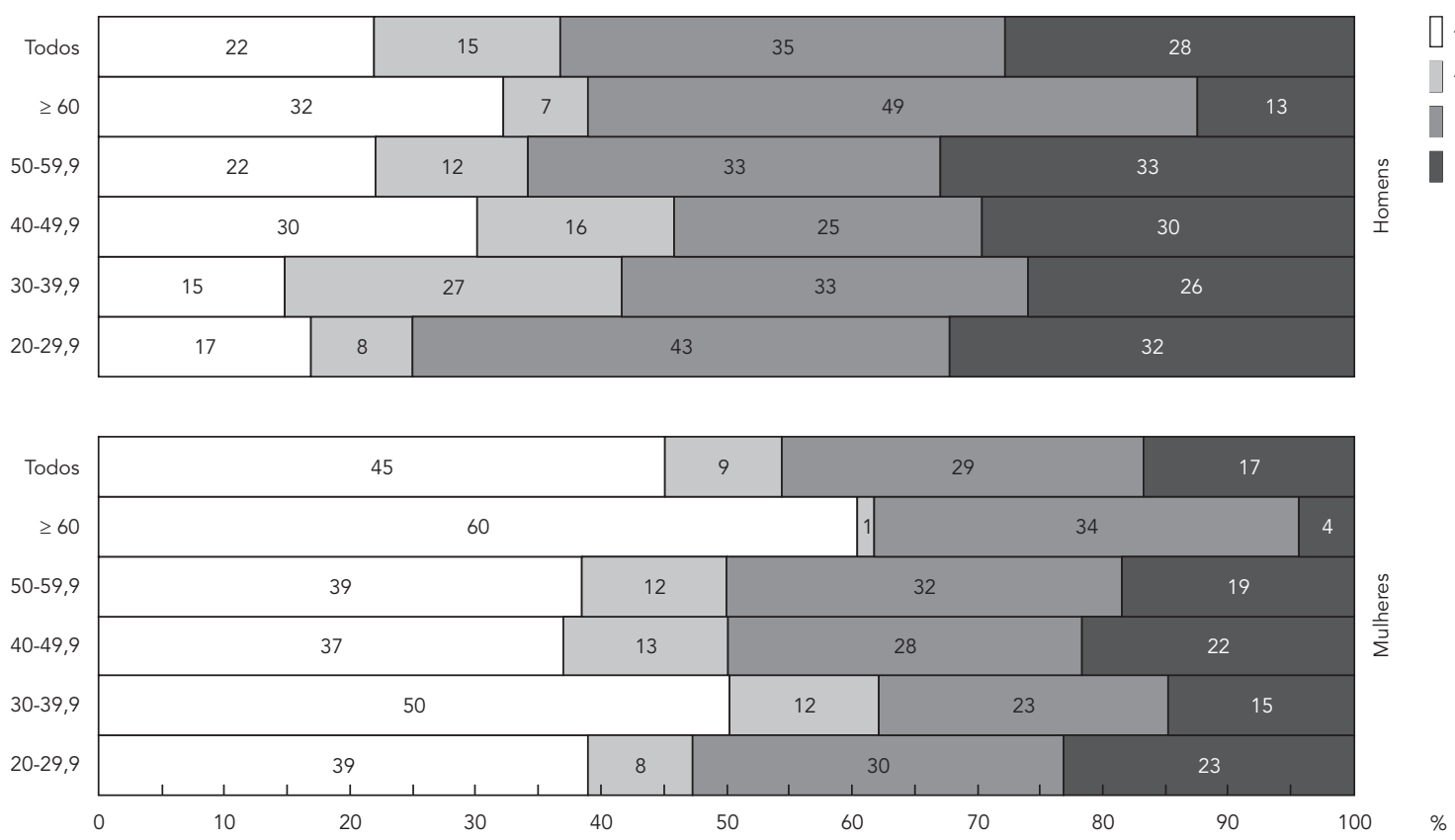

METs: múltiplos do equivalente metabólico. 
físico ou esporte ${ }^{33}$. Considerando o mesmo critério de classificação utilizado na PPV (somente realização de exercício físico ou esporte no lazer), a porcentagem de adultos inativos do presente estudo foi semelhante, mas reduziu substancialmente quando todos os domínios da atividade física foram incorporados à análise.

O fato de estimar a prevalência de inatividade física analisando somente a atividade física de lazer é causado pela dificuldade na obtenção de dados relacionados à ocupação e tarefas realizadas no domicílio. Entretanto, a classificação de um indivíduo ou uma população como inativa fisicamente, exige medição de todos os domínios de atividade realizada, pois os níveis de atividade física sofrerão influência das características comportamentais, culturais e econômicas de cada população ${ }^{5}$. Assim, indivíduos que não realizam atividades de lazer poderão ser considerados como inativos, no entanto, suas ocupações, atividades no lar ou formas de deslocamento podem representar grande parte das suas atividades físicas diárias 11 . Nesse aspecto, o Ministério da Saúde brasileiro vem, desde 2006, realizando um inquérito anual dentro de um sistema de monitoramento denominado Vigilância de Fatores de Risco e Proteção para Doenças Crônicas Não Transmissíveis de Inquérito Telefônico (VIGITEL). Os dados mais recentes, de 2010, indicaram que somente $14,2 \%$ da população eram inativos fisicamente computando-se o tempo livre, o deslocamento, o trabalho ou as atividades domésticas 34 .

A recomendação hegemônica atual para prevenção de doenças não transmissíveis na população mundial divulgada por organismos internacionais sugere que todos os indivíduos devam acumular pelo menos 30min de atividade física em intensidade moderada na maioria, preferencialmente todos os dias da semana ${ }^{3}$. Essa pode ser realizada em uma única sessão ou acumulada em curtas sessões ao longo do dia, como três sessões de $10 \mathrm{~min}$ ou duas de $15 \mathrm{~min}{ }^{30}$. O acúmulo das atividades em curtas sessões é mais atrativo e conveniente para aqueles indivíduos que julgam não ter tempo suficiente para realizar atividades físicas contínuas durante um período extenso, além da possibilidade de uma melhor tolerância por indivíduos sedentários que possuem menor nível de aptidão física 35 . Um outro fator bastante positivo é que essas atividades podem ser realizadas no lar, no trabalho ou em deslocamentos, não acarretando custo extra para os indivíduos, nem necessidade de aparatos ou equipamentos para sua realização. Apesar das facilidades apresentadas, ainda observa-se um alto percentual de pessoas que não alcançam esse nível de atividade. Considerando a atividade física em todos os seus domínios (ocupacional, doméstico, lazer e deslocamento), o presente trabalho revelou que $59,5 \%$ dos adultos da população $(63,8 \%$ homens e 55,9\% mulheres) de Niterói realizavam os 30min de atividade física propostos. Achado semelhante foi encontrado em estudo realizado em Pelotas, Rio Grande do Sul 36 , no qual $41,1 \%$ dos adultos foram classificados como inativos fisicamente. Embora os trabalhos tenham investigado a atividade física em sua totalidade, foram usados métodos diferentes (R24h vs. IPAQ), mas que, a princípio, investigam todos os domínios da atividade física 7 .

Estudos brasileiros que avaliam somente a atividade física de lazer têm mostrado que os homens são mais ativos fisicamente que as mulheres 34,37,38,39. Contudo, alguns estudos ao avaliar todos os domínios da atividade física têm mostrado resultados similaridades entre homens e mulheres 36,40. Mesmo tendo estudado a atividade física em todos os seus domínios, o presente trabalho encontrou que os homens são mais ativos fisicamente do que as mulheres, fato evidenciado pela maior permanência durante o dia em atividades de intensidades moderada e pesada. Um importante fator que contribui para a similaridade de padrão de atividade física encontrada entre homens e mulheres em alguns estudos é a inclusão das atividades domésticas, as quais em muitos casos são especificamente realizadas por mulheres 36 , mas que são particularmente difíceis de quantificar e determinar sua intensidade. De fato, as tarefas domésticas representaram, percentualmente, o dobro do tempo de atividade física moderada/pesada para mulheres do que para homens de Niterói. Usando um R24h, Dong et al. 18 identificaram maior percentual das atividades domésticas na contribuição do total do gasto energético diário das mulheres $(20,1 \%)$ do que dos homens $(33,3 \%)$ de amostra probabilística de adultos americanos no Inquérito sobre o Padrão de Atividade Humana (National Human Activity Pattern Survey). Matthews et al. 21, também documentaram percentuais maiores de contribuição das atividades domésticas em mulheres no total diário de gasto energético usando R24h. Ao detalhar as atividades em tempos menores e por recordar as atividades do dia anterior, o R24h pode captar tais atividades mais facilmente 18,21, o que, em estudos de sazonalidade, pode ser uma vantagem adicional.

Os resultados mostraram declínio na atividade física com o aumento da idade cronológica, fato já evidenciado na literatura 41 . Levando em consideração a realização de atividade física moderada/pesada durante o dia, observou-se, particularmente nos homens, tendência marcante de diminuição da atividade após os 40 anos. 
Considerando a atividade física moderada/ pesada e o domínio no qual foi realizada, observou-se que as atividades ocupacionais foram as que menos contribuíram para o total de atividade física tanto em homens quanto em mulheres, fato possivelmente explicado pela característica urbana da população de Niterói. Apesar de as tarefas ocupacionais serem de difícil modificação, por serem atividades já predefinidas e instituídas pelas próprias ocupações e, portanto, estarem se tornando cada vez mais sedentárias devido aos avanços tecnológicos, é possível que alguma modificação comportamental possa ser inserida no cotidiano dos trabalhadores. Algumas atitudes como subir e descer alguns lances de escada ao invés de usar o elevador ou fazer alguns intervalos para deambular, poderão contribuir para o aumento de atividades moderadas mesmo nas mais inativas das ocupações.

O presente estudo baseou-se na informação de atividade física de um único dia que, mesmo tendo sido caracterizado como típico pelos indivíduos, pode não representar o padrão de atividade física. Como em todo recordatório de 24h (alimentar ou de atividade física), os dados devem ser interpretados como representativos do padrão (alimentar ou de atividade física) da população como um todo. Apesar dessas limitações, o R24h permite o detalhamento das características da atividade física realizada (tipo duração e intensidade), o que nem sempre é fácil de se obter em outros questionários para os quais os sujeitos têm de fazer um esforço de estimação do tempo e frequência na realização de determinadas atividades num passado que pode ser recente ou remoto. Assim, o R24h é útil para a interpretação detalhada das atividades realizadas num intervalo de tempo curto, e estudos o tem utilizado para a documentação detalhada da atividade física em todos os domínios 18,21,22.

Uma outra questão inerente a qualquer questionário de atividade física diz respeito à codificação da intensidade das atividades realizadas. Como realizado em quase a totalidade de estudos da área, utilizou-se, no presente trabalho, o compêndio de atividade física ${ }^{29}$, cujos códigos de intensidade, apesar de largamente usados, ainda não foram devidamente validados. Pelo menos para a caminhada no plano em duas velocidades, os códigos do compêndio parecem ser adequados para a população adulta de Niterói 42,43 .

Os dados do presente estudo demonstraram que a maioria dos adultos residentes no Município de Niterói realiza atividade física suficiente para obtenção de benefícios à saúde e que, em geral, os homens realizam mais atividades moderadas e pesadas do que as mulheres. Mesmo assim, os resultados indicam a necessidade de um acréscimo diário de atividades fisicamente mais ativas no tempo livre e redução do tempo gasto em atividades leves.

\section{Resumo}

O objetivo do presente trabalho foi avaliar o padrão de atividade física em um dia típico de uma amostra probabilística de adultos $(N=1.689)$ de Niterói, Rio de Janeiro, Brasil, por meio de recordatório de atividade física de 24 horas. A amostra foi desenhada em três estágios (setor censitário, domicílio particular permanente e um adulto com equiprobabilidade, dentre os elegíveis, no domicílio selecionado). As atividades descritas foram classificadas em leves, moderadas e pesadas, e agrupadas em atividades domésticas, ocupacionais, no lazer e de deslocamento. Os homens realizam atividades moderadas e pesadas durante significativamente mais tempo $(91,2 \pm 5,6$ e $8,2 \pm 1,4 \mathrm{~min}$, respec- tivamente; média \pm erro padrão) do que as mulheres $(68,5 \pm 2,7$ e 2,9 90,4 min, respectivamente). Os percentuais de adultos que realizaram 30 min de atividades físicas moderadas/pesadas foram de 63,8\% e 55,9\% para homens e mulheres. As atividades domésticas das mulheres e o lazer ativo dos homens, foram os maiores contribuintes no total do tempo realizando atividades físicas moderadas/pesadas. Os dados demonstraram que a maioria dos adultos de Niterói é fisicamente ativa dentro do recomendado.

Atividade Motora; Atividades Cotidianas; Saúde do Adulto 


\section{Colaboradores}

L. A. Anjos supervisionou a coleta de dados, analisou os dados e revisou e aprovou a versão final do manuscrito. T. B. C. Barbosa participou da coleta de dados, escreveu uma versão inicial do texto e aprovou a versão final do manuscrito. V. Wahrlich supervisionou a coleta de dados e revisou e aprovou a versão final do manuscrito M. T. L. Vasconcellos fez o desenho de amostragem, calculou os pesos amostrais naturais e calibrados, realizou as análises e aprovou a versão final do manuscrito.

\section{Agradecimentos}

A Pesquisa de Nutrição, Atividade Física e Saúde (PNAFS) foi financiada em parte pelo Conselho Nacional de Desenvolvimento Científico e Tecnológico (CNPq, processos 471172/2001-4 e 475122/2003-8) e pela Fundação Oswaldo Cruz (Fiocruz: PAPES III - Programa de Apoio a Projetos Estratégicos em Saúde - 250.139). L. A. Anjos recebeu bolsa de produtividade em pesquisa do CNPq (processos 302952/2003-9, 311801/2006-4 e 308489/2009-8). M. T. L. Vasconcellos recebeu bolsa de produtividade em pesquisa do CNPq (processo 302992/2003-0).

\section{Referências}

1. World Health Organization. Preventing chronic diseases: a vital investment: WHO global report. Geneva: World Health Organization; 2005.

2. Haskell WL, Blair SN, Hill JO. Physical activity: health outcomes and importance for public health policy. Prev Med 2009; 49:280-2.

3. Haskell WL, Lee IM, Pate RR, Powell KE, Blair SN, Franklin BA, et al. Physical Activity and public health: updated recommendation for adults from the American College of Sports Medicine and the American Heart Association. Med Sci Sports Exerc 2007; 39:1423-34.

4. U.S. Department of Health and Human Services Dietary guidelines for Americans. Washington DC: U.S. Department of Health and Human Services; 2005.

5. Anjos LA, Wahrlich V. Gasto energético: medição e importância para a área de nutrição. In: Kac G, Sichieri R, Gigante DP, organizadores. Epidemiologia nutricional. Rio de Janeiro: Editora Fiocruz/ Editora Atheneu; 2007. p. 165-80.

6. Montoye HJ, Kemper HCG, Saris WHM, Washburn RA. Measuring physical activity and energy expenditure. Champaign: Human Kinetics; 1996.

7. Laporte RE, Montoye HJ, Caspersen CJ. Assessment of physical activity in epidemiologic research: problems and prospects. Public Health Rep 1985; 100:131-46.

8. Pitanga FJG, Lessa I. Prevalência e fatores associados ao sedentarismo no lazer em adultos. Cad Saúde Pública 2005; 21:870-7.

9. Dias-da-Costa JS, Hallal PC, Wells JCK, Daltoé T, Fuchs SC, Menezes AMB, et al. Epidemiology of leisure-time physical activity: a population-based study in southern Brazil. Cad Saúde Pública 2005; 21:275-82.
10. U.S. Department of Health and Human Services; Centers for Disease Control and Prevention; National Center for Chronic Disease Prevention and Health Promotion; The President's Council on Physical Fitness and Sports. Physical activity and health: a report of the Surgeon General. Executive summary. Atlanta: Centers for Disease Control and Prevention; 1996.

11. Centers for Disease Control and Prevention. Prevalence of leisure-time and occupational physical activity among employed adults - United States, 1990. MMW Rep 2000; 49:420-4.

12. Craig CL, Marshall AL, Sjöström M, Bauman AE, Booth ML, Ainsworth BE, et al. International Physical Activity Questionnaire: 12-country reliability and validity. Med Sci Sports Exerc 2003; 35: 1381-95.

13. Maddison R, Ni Mhurchu C, Jiang Y, Vander Hoorn S, Rodgers A, Lawes CM, et al. International Physical Activity Questionnaire (IPAQ) and New Zealand Physical Activity Questionnaire (NZPAQ): a doubly labelled water validation. Int J Behav Nutr Phys Act 2007; 4:62.

14. Hallal PC, Simões E, Reichert FF, Azevedo MR, Ramos LR, Pratt M, et al. Validity and reliability of the telephone-administered International Physical Activity Questionnaire in Brazil. J Phys Act Health 2010; 7:402-9.

15. Loney T, Standage M, Thompson D, Sebire SJ, Cumming S. Self-report vs. objectively assessed physical activity: Which is right for public health? J Phys Act Health 2011; 8:62-70. 
16. Lee PH, Yu YY, McDowell I, Leung GM, Lam TH, Stewart SM. Performance of the International Physical Activity Questionnaire (short form) in subgroups of the Hong Kong Chinese population. Int J Behav Nutr Phys Act 2011; 8:81.

17. Lee PH, Macfarlane DJ, Lam TH, Stewart SM. Validity of the International Physical Activity Questionnaire Short Form (IPAQ-SF): a systematic review. Int J Behav Nutr Phys Act 2011; 8:115.

18. Dong L, Block G, Mandel S. Activities contributing to total energy expenditure in the United States: Results from the NHAPS Study. Int J Behav Nutr Phys Act 2004; 1:4.

19. Food and Agriculture Organization. Human energy requirements. Report of a Joint $\mathrm{FAO} / \mathrm{WHO} /$ UNU Expert Consultation. Rome: Food and Agriculture Organization of the United Nations; 2004. (FAO Technical Report Series, 1).

20. Calabro MA, Welk GJ, Carriquiry AL, Nusser SM, Beyler NK, Matthews CE. Validation of a computerized 24-hour physical activity recall (24PAR) instrument with pattern-recognition activity monitors. J Phys Act Health 2009; 6:211-20.

21. Matthews CE, Freedson PS, Hebert JR, Stanek EJ Merriam PA, Ockene IS. Comparing physical activity assessment methods in the Seasonal Variation of Blood Cholestherol Study. Med Sci Sports Exerc 2000; 32:976-84.

22. Ma Y, Olendzki BC, Li W, Hafner AR, Chiriboga D, Hebert JR, et al. Seasonal Variation in food intake, physical activity, and body weight in predominantly overweight population. Eur J Clin Nutr 2006; 60:519-28.

23. Bossan FM, Anjos LA, Vasconcellos MTL, Wahrlich $\mathrm{V}$. Nutritional status of the adult population in $\mathrm{Ni}$ terói, Rio de Janeiro, Brazil: the Nutrition, Physical Activity, and Health Survey. Cad Saúde Pública 2007; 23:1867-76.

24. Instituto Brasileiro de Geografia e Estatística. Resultados da amostra do Censo Demográfico 2000: malha municipal digital do Brasil: situação em 2001. Rio de Janeiro: Instituto Brasileiro de Geografia e Estatística; 2004.

25. Haldane JBS. On a method of estimating frequencies. Biometrika 1945; 33:222-5.

26. Vasconcellos MTL, Silva PLN, Szwarcwald CL Sampling design for the World Health Survey in Brazil. Cad Saúde Pública 2005; 21 Suppl 1:S89-99.

27. Silva PLN. Calibration estimation: when and why how much and how. Rio de Janeiro: Instituto Brasileiro de Geografia e Estatística; 2004. (Textos para Discussão da Diretoria de Pesquisas, 14).

28. Souza DR, Anjos LA, Wahrlich V, Vasconcellos MTL Machado JM. Ingestão alimentar e balanço energético da população adulta de Niterói, Rio de Janeiro, Brasil: resultados da Pesquisa de Nutrição, Atividade Física e Saúde (PNAFS). Cad Saúde Pública 2010; 26:879-90.

29. Ainsworth BE, Haskell WL, Whitt MC, Irwin ML, Swartz AM, Strath SJ, et al. Compendium of physical activities: an update of activity codes and MET intensities. Med Sci Sports Exerc 2000; 32 (9 Suppl):S498-516.
30. Pate RR, Pratt M, Blair SN, Haskell WL, Macera CA, Bouchard C, et al. Physical activity and public health: a recommendation from the centers for disease control and prevention and the American College of Sports Medicine. JAMA 1995; 273:402-7.

31. Dumith SC. Physical activity in Brazil: a systematic review. Cad Saúde Pública 2009; 25 Suppl 3: S415-26.

32. Hallal PC, Dumith SC, Bastos JP, Reichert FF, Siqueira FV, Azevedo MR. Evolução da pesquisa epidemiológica em atividade física no Brasil: revisão sistemática. Rev Saúde Pública 2007; 41:453-60.

33. Mendonça CP, Anjos LA. Aspectos das práticas alimentares e da atividade física como determinantes do crescimento do sobrepeso/obesidade no Brasil. Cad Saúde Pública 2004; 20:698-709.

34. Secretaria de Vigilância em Saúde/Secretaria de Gestão Estratégica e Participativa, Ministério da Saúde. VIGITEL Brasil 2010: vigilância de fatores de risco e proteção para doenças crônicas por inquérito telefônico. Brasília: Ministério da Saúde; 2011.

35. Staffileno BA, Braun LT, Rosenson RS. The accumulative effects of physical activity in hypertensive post-menopausal women. J Cardiovasc Risk 2001; 8:283-90.

36. Hallal PC, Victoria CG, Wells JCK, Lima RC. Physical inactivity: prevalence and associated variables in Brazilian adults. Med Sci Sports Exerc 2003; 35:1894-900

37. Barros MVG, Nahas MV. Comportamentos de risco, auto-avaliação do nível de saúde e percepção de estresse entre trabalhadores da indústria. Rev Saúde Pública 2001; 35:554-63.

38. Gomes VB, Siqueira KS, Sichieri R. Atividade física em uma amostra probabilística da população do Município do Rio de Janeiro. Cad Saúde Pública 2001; 17:969-76.

39. Salles-Costa R, Werneck GL, Lopes CS, Faerstein E. Associação entre fatores sócio-demográficos e prática de atividade física de lazer no Estudo PróSaúde. Cad Saúde Pública 2003; 19:1095-105.

40. Matsudo SM, Matsudo VR, Araújo T, Andrade D, Andrade E, Oliveira L, et al. Nível de atividade física da população do Estado de São Paulo: análise de acordo com o gênero, idade, nível socioeconômico, distribuição geográfica e de conhecimento. Rev Bras Ciênc Mov 2002; 10:41-50.

41. Burton NW, Turrell G. Occupation, hours worked, and leisure-time physical activity. Prev Med 2000; 31:673-81.

42. Anjos LA, Wahrlich V, Bossan FM, Salies MN, Silva PB. Energy expenditure of walking at different intensities in Brazilian college women. Clin Nutr 2008; 27:121-5.

43. Anjos LA, Machado JM, Wahrlich V, Vasconcellos MTL, Caspersen CJ. Absolute and relative energetic costs of walking in a Brazilian adult probability sample. Med Sci Sports Exerc 2011; 43:2211-8.

Recebido em 27/Out/2011

Versão final reapresentada em 02/Jul/2012

Aprovado em 06/Jul/2012 